\title{
ON SOME SUB-RIEMANNIAN OBJECTS IN HYPERSURFACES OF SUB-RIEMANNIAN MANIFOLDS
}

\author{
Kang-HaI TAN AND XIAO-PING YANG
}

\begin{abstract}
We study some sub-Riemannian objects (such as horizontal connectivity, horizontal connection, horizontal tangent plane, horizontal mean curvature) in hypersurfaces of sub-Riemannian manifolds. We prove that if a connected hypersurface in a contact manifold of dimension more than three is noncharacteristic or with isolated characteristic points, then there exists at least a piecewise smooth horizontal curve in this hypersurface connecting any two given points in it. In any sub-Riemannian manifold, we obtain the sub-Riemannian version of the fundamental theorem of Riemannian geometry: there exists a unique nonholonomic connection which is conpletely determined by the sub-Riemannian structure and is "symmetric" and compatible with the sub-Riemannian metric. We use this nonholonomic connection to study horizontal mean curvature of hypersurfaces.
\end{abstract}

\section{INTRODUCTION}

Recently there is an explosion of interest in the theory of sub-Riemannian manifolds (or Carnot-Carathéodory spaces in general), and in the ramifications of this subject in analysis and geometry, see for example, $[1,5,6,9,10,11,12,13,16,19,22]$. We recall a sub-Riemannian manifold $\left(M, \Delta, g_{c}\right)$ is a smooth manifold $M$ with a distribution $\Delta(\mathrm{a}$ subbundle of the tangent bundle $T M$ ) which is endowed with a fibrewise inner product $g_{c}$ (usually called the sub-Riemannian metric). $g_{c}$ is usually realised as the restriction on $\Delta$ of some Riemannian metric $g$ on $T M$. Carnot groups are particularly interesting subRiemannian manifolds. Roughly speaking, the sub-Riemannian geometry of $\left(M, \Delta, g_{c}\right)$ is the geometry determined by the sub-Riemannian structure $\left(\Delta, g_{c}\right)$ which yields CarnotCarathéodory distance if $\Delta$ satisfies Chow's condition and $M$ is connected, and should be independent of the choice of the Riemannian metric $g$ which is the extension of $g_{c}$ to $T M$, although it often interacts with the Riemannian geometry of $(M, g)$.

It is natural to study the geometry of hypersurfaces (or submanifolds of codimension more than one) in sub-Riemannian manifolds not only from the geometric viewpoint (see $[5,13,14,22]$ which study sub-Riemannian minimal surfaces in Carnot groups), but also from the viewpoint of analysis (see $[9,10,11,12,19,23]$ for the development

Received 13th October, 2003

Copyright Clearance Centre, Inc. Serial-fee code: 0004-9727/04 \$A2.00+0.00. 
of geometric measure theory in the setting of sub-Riemannian geometry). To develop a theory of sub-Riemannian minimal surfaces, a sub-Riemannian counterpart of the notion of the usual mean curvature on submanifolds should be laid down. In [22, 5] (see also [14]) an analysis definition of the notion of horizontal mean curvature for noncharacteristic hypersurfaces in Carnot groups has been given. In [26] we have formulated a geometric definition of the horizontal mean curvature by using the notion of horizontal connection. It turns out that our definition coincides with the analysis definition in the case of Carnot groups. The geometric definition is valid for general sub-Riemannian manifolds.

In this paper we continue our study of the geometry and calculus of hypersurfaces in sub-Riemannian manifolds. The notion of the horizontal tangent plane of smooth noncharacteristic (see Definition 3.1) hypersurfaces plays an important role in the development of [26]. The horizontal tangent plane $T_{x}^{\mathcal{H}} S$ at a point $x$ in a smooth noncharacteristic hypersurface $S$ is defined as the $k-1$ (assuming $\Delta$ is $k$ dimensional) dimensional linear space $T_{x}^{\mathcal{H}} S$ such that

$$
\Delta_{x}=n^{\mathcal{H}}(x) \bigoplus^{g_{c}} T_{x}^{\mathcal{H}} S
$$

where $\Delta_{x}$ is the fibre of $\Delta$ through $x, n^{\mathcal{H}}(x)$ the horizontal normal of $S$ at $x$ and $\stackrel{g_{c}}{\bigoplus}$ denotes the orthogonal decomposition with respect to $g_{c}$. Since $n^{\mathcal{H}}(x)$ is by definition the projection onto $\Delta_{x}$ of the Riemannian normal $n(x)$ computed with respect to $g$ (any orthogonal extension of $g_{c}$ ), we have

$$
T_{x}^{\mathcal{H}} S=T_{x} S \cap \Delta_{x}
$$

We call

$$
T^{\mathcal{H}} S:=\bigcup_{x \in S} T_{x}^{\mathcal{H}} S
$$

the horizontal tangent bundle. In general, if $S$ possesses characteristic points, one also can define $T^{\mathcal{H}} S=T S \cap \Delta$. Note that $T^{\mathcal{H}} S$ is independent of the orthogonal extensions of $g_{c}$. A very interesting question is under what conditions $T^{\mathcal{H}} S$ satisfies the Chow's condition with respect to $T S$, that is, the subbundle $T^{\mathcal{H}} S$ (if it is a subbundle, say) together with all its Lie commutators spans $T S$ (we assume $\Delta$ satisfies the Chow's condition). This question is relevant to another two: whether there exist sufficiently many horizontal curves in $S$ and whether one can define a Carnot-Carathéodory metric with respect to $T^{\mathcal{H}} S$ with an induced metric $g_{c}^{\mathcal{H}}$ (from $g_{c}$ )? We point out that in his seminal paper [16] Gromov discussed, (along with other things about geometry of hypersurfaces in sub-Riemannian manifolds), the relationship between the restricted Carnot-Caratheodory metric on $S$ and the Carnot-Carathéodory metric induced by the structure $\left(T^{\mathcal{H}} S, g_{c}^{\mathcal{H}}\right)$. He claimed that the two metrics are Lipschitz equivalent in some special cases, for example, contact manifolds of dimension more than tree, see page 104 and page 173 in [16]. One of the main results of this paper is to give the first result in this regard. We have 
THEOREM 1.1. Let $M$ be a smooth orientable contact manifold of dimension $2 n+1$ with a contact form $\eta$ and a Riemannian metric $g$ and let $\Delta:=\operatorname{ker}(\eta)$ be the canonical distribution. Let $S$ be a smooth connected hypersurface in $M$. If $n>1$ and there does not exist an immersed submanifold contained in the set $\Sigma_{S}$ of all characteristic points in $S$, then for any points $p, q \in S$, there exists a piecewise smooth curve $\gamma(t)$, $t \in[a, b]$ connecting $p, q$ such that $\dot{\gamma} \in T_{\gamma(t)}^{\mathcal{H}} S$. In particular, if $S$ is noncharacteristic, $T^{\mathcal{H}} S$ as a subbundle of TS satisfies the Chow's condition, that is, $T^{\mathcal{H}} S$ together with all its Lie commutators span TS.

Theorem 1.1 follows from Sussmann's famous Orbit Theorem and the contact structure of $M$. A trivial example shows that the condition of dimension more than three is necessary.

The next point we concentrate on is whether there exists an intrinsic nonholonomic connection $D$ in $\left(M, \Delta, g_{c}\right)$. Here by "intrinsic" we mean that it is completely determined by the sub-Riemannian structure $\left(\Delta, g_{c}\right)$. A natural candidate is the one $\widetilde{D}$ obtained by projecting to $\Delta$ the Levi-Civita connection $\nabla$ with respect to some orthogonal extension $g$ of $g_{c}$. We prove that $\widetilde{D}$ is independent of the choice of orthogonal extension of $g_{c}$. Moreover we have

THEOREM 1.2. Let $\left\{X_{1}, \ldots, X_{k}\right\}$ be an orthonormal basis of $\Delta$. We define $D$ by

$$
\begin{aligned}
D_{U} V & =\sum_{i=1}^{k} U\left(V^{i}\right) X_{i}+\sum_{i=1}^{k} \sum_{j=1}^{k} \sum_{l=1}^{k} U^{j} V^{i} \Gamma_{i j}^{l} X_{l} \text { for any } \\
U & =\sum_{j=1}^{k} U^{j} X_{j}, \quad V=\sum_{i=1}^{k} V^{i} X_{i} \in \Gamma(\Delta)
\end{aligned}
$$

where

$$
\Gamma_{i j}^{l}=-\frac{1}{2}\left\{g_{c}\left(X_{i},\left[X_{j}, X_{l}\right]^{\mathcal{H}}\right)+g_{c}\left(X_{j},\left[X_{l}, X_{i}\right]^{\mathcal{H}}\right)-g_{c}\left(X_{l},\left[X_{i}, X_{j}\right]^{\mathcal{H}}\right)\right\}
$$

and $X^{\mathcal{H}}$ is understood as the projection of $X$ to $\Delta$ with respect to the direct summation decomposition $T M=\Delta \oplus \widetilde{\Delta}$ where $\widetilde{\Delta}$ is the distribution complementary to $\Delta$. Then $D$ is independent of the choice of orthonormal basis of $\Delta$ and it is the unique nonholonomic connection satisfying

(1) $D_{U} V$ is $R$-linear in both arguments,

(2) $D_{U} V$ is $C^{\infty}(M)$-linear in the argument of $U$,

(3) the Leibniz rule holds:

$D_{U}(f V)=(U f) V+f D_{U} V$ for any $f \in C^{\infty}(M), U, V \in \Gamma(\Delta)$,

(4) $D$ is compatible with respect to $g_{c}$, that is, 


$$
U g_{c}(V, W)=g_{c}\left(D_{U} V, W\right)+g_{c}\left(U, D_{V} W\right) \text { for any } U, V, W \in \Gamma(\Delta)
$$

(5) the following "symmetry" property holds:

$$
D_{U} V-D_{V} U=[U, V]^{\mathcal{H}} \text { for any } U, V \in \Gamma(\Delta) \text {. }
$$

In particular we have $D=\widetilde{D}$.

Theorem 1.2 is the counterpart of the existence and uniqueness of the Levi-Civita connection in Riemannian geometry.

We shall use $D$ to study the horizontal mean curvature of hypersurfaces in $\left(M, \Delta, g_{c}\right)$. Let $D^{\top}$ be the tangent horizontal connection on the horizontal tangent bundle $T^{\mathcal{H}} S$ of a smooth noncharacteristic hypersurface $S$. We define the horizontal mean curvature of $S$ as the trace of the horizontal fundamental second form which is by definition a bilinear map II from $\Gamma\left(T^{\mathcal{H}} S\right) \times \Gamma\left(T^{\mathcal{H}} S\right)$ to $N$ :

$$
\mathrm{II}(X, Y)=D_{X} Y-D_{X}^{\top} Y
$$

for any $X, Y \in \Gamma\left(T^{\mathcal{H}} S\right)$ where $\Gamma\left(T^{\mathcal{H}} S\right)$ denotes all smooth sections of $T^{\mathcal{H}} S$ and $N$ is the horizontal normal. The symmetry of II follows from the symmetry property (1.3) of $D$ and the definition of the horizontal tangent plane. Since both $D$ and $T^{\mathcal{H}} S$ are intrinsic, so is the horizontal mean curvature.

The paper is organised as follows. In Section 2 we collect some facts about subRiemannian manifolds which will be used later, mainly to fix some notations. In Section 3 Theorem 1.1 is proved after introducing the notion of the horizontal tangent bundle. Roughly speaking, if we project Riemannian objects onto the horizontal bundle (such as the Riemannian connection, normal vector and tangent bundle), then we get corresponding sub-Riemannian analogues: horizontal connection, horizontal normal vector and horizontal bundle. Theorem 1.2 is proved in Section 4.

\section{BASIC MATERIAL ON SUB-RIEMANNIAN MANIFOLDS}

Let $M$ be a smooth $\left(C^{\infty}\right)$ manifold of dimension $m$ endowed with a smooth distribution (called the horizontal bundle) $\Delta$ of dimension $k$ with $k<m$. [Note that this imposes topological constraints on $M$, see [18].] If we a priori equip $\Delta$ with an inner product $g_{c}$ (called the sub-Riemannian metric), we call $\left(M, \Delta, g_{c}\right)$ a sub-Riemannian manifold with the sub-Riemannian structure $\left(\Delta, g_{c}\right)$. Let $\left\{X_{1}, \ldots, X_{k}\right\}$ be an orthonormal basis of $\Delta$. [If a global basis does not exist, we can consider a local basis (which always exists) without restriction for our purposes.] A piecewise smooth curve $\gamma(t), t \in[a, b]$ in $M$ is horizontal if $\dot{\gamma}(t) \in \Delta_{\gamma(t)}$ almost everywhere $t \in[a, b]$. The length $\ell(\gamma)$ of the horizontal 
curve $\gamma(t), t \in[a, b]$ is the integral $\int_{a}^{b} g_{c}(\dot{\gamma}(t), \dot{\gamma}(t)) d t$. Denote by $L_{i}$ the set of all vector fields spanned by all commutators of $X_{j}$ 's of order $\leqslant i$ and let $L_{i}(p)$ be the subspace of evaluations at $p$ of all vector fields in $L_{i}$. We say $\Delta$ satisfies Chow's condition [in the subelliptic theory, Chow's condition is also called Hörmander's condition] if for any $p \in M$, there exists an integer $r(p)$ such that $L_{r(p)}(p)=T_{p} M$ (the tangent space of $M$ at $p$ ). If $M$ is connected and $\Delta$ satisfies Chow's condition, the Chow connectivity theorem asserts that there exists at least one piecewise smooth horizontal curve connecting two given points (see $[4, \mathbf{1}]$ or $[\mathbf{1 6}]$ ), and thus $\left(\Delta, g_{c}\right)$ yields a metric (called the Carnot-Carathéodory metric) $d_{c}$ by letting $d_{c}(p, q)$ be the infimum among the lengths of all horizontal curves joining $p$ to $q$.

$\Delta$ is equiregular if the dimension of $L_{i}(p)$ does not depend on $p$ for any $i$, that is, the tangent bundle $T M$ is filtered by smooth subbundles

$$
\Delta=L_{1} \subset L_{2} \subset \cdots \subset L_{r}=T M
$$

where $r$ is called the degree of $\Delta$. Note that we can always extend $g_{c}$ to a Riemannian metric $g$ in $M$ such that $T M$ can be $g$-orthogonally decomposed as $T M=\Delta \stackrel{g}{\oplus} \widetilde{\Delta}$ where $\tilde{\Delta}$ is the distribution complementary to $\Delta$. We call such $g$ an orthogonal extension of $g_{c}$. Obviously the orthogonal extension of $g_{c}$ is not unique in general. We shall use $\Gamma(\Delta)$ to denote the set of all smooth sections of $\Delta$.

EXAMPLE 2.1. (Carnot groups) The most interesting models of sub-Riemannian manifolds are Carnot groups (called also stratified groups). A Carnot group $G$ is a connected, simply connected Lie group whose Lie algebra $\mathcal{G}$ admits the grading $\mathcal{G}=V_{1} \oplus \cdots \oplus V_{l}$, with $\left[V_{1}, V_{i}\right]=V_{i+1}$, for any $1 \leqslant i \leqslant l-1$ and $\left[V_{1}, V_{l}\right]=0$ (the integer $l$ is called the step of $G$ ). Let $\left\{e_{1}, \ldots, e_{n}\right\}$ be a basis of $\mathcal{G}$ with $n=\sum_{i=1}^{l} \operatorname{dim}\left(V_{i}\right)$. Let $X_{i}(g)=\left(L_{g}\right)_{*} e_{i}$ for $i=1, \ldots, k:=\operatorname{dim}\left(V_{1}\right)$ where $\left(L_{g}\right)$. is the differential of the left translation $L_{g}\left(g^{\prime}\right)=g g^{\prime}$ and let $Y_{i}(g)=\left(L_{g}\right)_{*} e_{i+k}$ for $i=1, \ldots, n-k$. We call the system of left-invariant vector fields $\Delta:=V_{1}=\operatorname{span}\left\{X_{1}, \ldots, X_{k}\right\}$ the horizontal bundle of $G$. If we equip $\Delta$ an inner product $g_{c}$ such that $\left\{X_{1}, \ldots, X_{k}\right\}$ is an orthonormal basis of $\Delta,\left(G, \Delta, g_{c}\right)$ is an equiregular sub-Riemannian manifold. In $\left(G, \Delta, g_{c}\right), d_{c}$ is invariant with respect to left translation, that is $d_{c}\left(p_{0} p, p_{0} q\right)=d_{c}(p, q)$ for any $p_{0}, p, q \in G$, and is 1-homogeneous with respect to the natural dilations, that is $d_{c}\left(\delta_{s} p, \delta_{s} q\right)=s d_{c}(p, q)$ for any $s>0, p, q \in G$, where $\delta_{s} p=\exp \left(\sum_{i=1}^{l} s^{i} \xi_{i}\right)$ for $p=\exp \left(\sum_{i=1}^{l} \xi_{i}\right), \xi_{i} \in V_{i}$.

EXAMPLE 2.2. (Contact manifolds) Contact manifolds are also interesting subRiemannian manifolds not necessarily with group structure. A contact manifold is a smooth (connected) manifold $M$ with a contact structure which is by definition a codimension one distribution $\Delta \subset T M$ with non-degenerate curvature form $\omega: \Delta \wedge \Delta$ $\rightarrow T M / \Delta$ which is defined as follows: represent $\Delta$ locally as the kernel of a 1-form, say $\eta$ 
on $M$, identify $T M / \Delta$ with the trivial line bundle and then define $\omega$ as $d \eta \mid \Delta . \eta$ is called a contact form of $M$ (not unique). If $M$ is orientable, $\eta$ and $\omega$ can be globally defined. The non-degeneracy of $\omega$ makes the dimension of $\Delta$ to be even and so the dimension of $M$ is odd, say $2 n+1$. Note that the non-vanishing of $\omega$ on $\Delta$ makes the commutators of degree $\leqslant 2$ span $T M$ and thus $\left(M, \Delta, g_{c}\right)$ is an equiregular sub-Riemannian manifold where $g_{c}$ is the restriction on $\Delta$ of some Riemannian metric $g$ on $M$.

EXAMPLE 2.3. (Heisenberg group) The Heisenberg group $\mathbb{H}^{n}$ as a representative both in the class of Carnot groups and in the class of contact manifolds is of paramount importance, and is worthy of being paid more attention. The underlying manifold of this Lie group is simply $\mathbb{R}^{2 n+1}$, with the noncommutative group law

$$
p \cdot p^{\prime}=(x, y, t) \cdot\left(x^{\prime}, y^{\prime}, t^{\prime}\right)=\left(x+x^{\prime}, y+y^{\prime}, t+t^{\prime}+2\left(\left\langle x^{\prime}, y\right\rangle-\left\langle x, y^{\prime}\right\rangle\right)\right)
$$

where we have let $x, x^{\prime}, y, y^{\prime} \in \mathbb{R}^{n}, t, t^{\prime} \in \mathbb{R}$. A simple computation shows that

$$
\begin{aligned}
X_{j}(p) & \stackrel{\text { def }}{=}\left(L_{p}\right)_{*}\left(\frac{\partial}{\partial x_{j}}\right)=\frac{\partial}{\partial x_{j}}+2 y_{j} \frac{\partial}{\partial t}, \quad j=1, \ldots, n, \\
X_{n+j}(p) & \stackrel{\text { def }}{=}\left(L_{p}\right)_{*}\left(\frac{\partial}{\partial y_{j}}\right)=\frac{\partial}{\partial y_{j}}-2 x_{j} \frac{\partial}{\partial t}, \quad j=1, \ldots, n, \\
T(p) & \stackrel{\text { def }}{=}\left(L_{p}\right)_{*}\left(\frac{\partial}{\partial t}\right)=\frac{\partial}{\partial t}
\end{aligned}
$$

for any $p=(x, y, t)$ in $\mathbb{H}^{n}$. We note that

$$
\begin{aligned}
& {\left[X_{j}, X_{n+k}\right]=-4 T \delta_{j k}, \quad j, k=1, \ldots, n,} \\
& \text { and all other commutators are trivial, }
\end{aligned}
$$

therefore the vector fields $X=\left\{X_{1}, \ldots, X_{2 n}\right\}$ constitute a basis of the Lie algebra $b_{n}=\mathbb{R}^{2 n+1}=V_{1} \oplus V_{2}$, where $V_{1}=\mathbb{R}^{2 n} \times\{0\}_{t}, V_{2}=\{0\}_{x, y} \times \mathbb{R}$. Note that the horizontal bundle $\Delta=\operatorname{span}\left\{X_{1}, \ldots, X_{2 n}\right\}$ is the kernel of the 1-form $\eta=(d t / 4)+\left(\sum_{i=1}^{n} / 2\right)\left(x_{i} d y_{i}\right.$ $\left.-y_{i} d x_{i}\right)$ and the curvature form $\omega=d \eta=\sum_{i=1}^{n} d x_{i} \wedge d y_{i}$ is the standard symplectic form in $\mathbb{R}^{2 n}$. Thus a smooth curve $\gamma(s)=(x(s), y(s), t(s)):[a, b] \rightarrow \mathbb{H}^{n}$ is horizontal if and only if

$$
2 \dot{t}(s)=\sum_{i=1}^{n} y_{i}(s) \dot{x}_{i}(s)-x_{i}(s) \dot{y}_{i}(s) \quad \text { for any } s \in[a, b] .
$$

For the theory of sub-Riemannian geodesics we refer to the book [21] and references therein. See [16] for a comprehensive treatment of the geometry (more than subRiemannian geodesics) in sub-Riemannian manifolds, and for many potential research directions. 


\section{ThE hORIZONTAL TANGENT BUNDLE AND HORIZONTAL CONNECTIVITY IN HYPERSURFACES}

Let $\left(M, \Delta, g_{c}\right)$ be a sub-Riemannian manifold. In this section we always assume $M$ is connected and $\Delta$ satisfies Chow's condition. By $S$ we always mean a smooth $\left(C^{\infty}\right)$ hypersurface (that is, a embedded submanifold of codimension 1) in $M$. Let $g$ be any orthogonal extension of $g_{c}$.

Definition 3.1: (Characteristic points) A point $p \in S$ is a characteristic point if $\Delta_{p} \subset T_{p} S$. Let $\Sigma_{S}$ denote the set of all characteristic points in $S$. If $\Sigma_{S}=\emptyset$, we say $S$ is noncharacteristic.

Typically $S$ possesses characteristic points, see for example, [6]. But we have

Proposition 3.2. $\Sigma_{S}$ is a closed subset of $S$, and $\mathcal{H}^{m-1}\left(\Sigma_{S}\right)=0$. Here $\mathcal{H}^{m-1}$ denotes the $m-1$ dimensional Hausdorff measure with respect to the Riemannian metric $g$ (recall $M$ is $m$-dimensional).

The closedness follows from the smoothness of $S$ and the smallness of $\Sigma_{S}$ is due to Derridj, see [7, 8]. In the case of Carnot groups of step two, when the smoothness of $S$ is weaker than $C^{\infty}$, say $C^{2}$, the smallness of $\Sigma_{S}$ is obtained by Magnani, see [19, 20].

By Proposition 3.2, if $p \in S \backslash \Sigma_{S}$, then there exists a neighbourhood $\mathcal{U}$ of $p$, such that $\mathcal{U} \cap S \subset S \backslash \Sigma_{S}$.

Definition 3.3: (Horizontal normal) Let $n^{g}$ denote the Riemannian normal of $S$ with respect to $g$. We define the horizontal normal $n^{\mathcal{H}}$ of $S$ as the projection of the Riemannian normal onto the horizontal bundle, that is,

$$
n^{\mathcal{H}}=\sum_{i=1}^{k} g\left(n^{g}, X_{i}\right) X_{i}
$$

where $\left\{X_{1}, \ldots, X_{k}\right\}$ is an orthonormal basis of $\Delta$.

It is easily seen that $p \in S$ is a characteristic point if and only if $n^{\mathcal{H}}(p)=0$.

Definition 3.4: (Horizontal tangent plane) For $p \in S$, we define

$$
T_{p}^{\mathcal{H}} S:=\left\{v \in \Delta_{p}: g_{c}\left(v, n^{\mathcal{H}}(p)\right)=0\right\}
$$

as the horizontal tangent plane of $S$ at $p$.

From the definition we see that if $p \in \Sigma_{S}$ then $T_{p}^{\mathcal{H}} S=\Delta_{p}$, and otherwise $T_{p}^{\mathcal{H}} S$ is a $k-1$ dimensional subspace of $\Delta_{p}$. Thus

$$
T^{\mathcal{H}} S:=\bigcup_{p \in S} T_{p}^{\mathcal{H}} S
$$

is a distribution of dimension $k-1$ if and only if $S$ is noncharacteristic. The following proposition shows that $T^{\mathcal{H}} S$ depends only on $\Delta$ and $S$. 
PROPOSITION 3.5. The horizontal tangent bundle $T^{\mathcal{H}} S$ is intrinsic, that is

$$
T^{\mathcal{H}} S=\Delta \cap T S
$$

in the sense that $T_{p}^{\mathcal{H}} S=\Delta_{p} \cap T_{p} S$ for any $p \in S$.

PROOF: Let $g$ be any orthogonal extension of $g_{c}$. For $p \in S$, first let $v \in T_{p}^{\mathcal{H}} S$. Thus $v \in \Delta_{p}$ and from Definition 3.3 we have

$$
\begin{aligned}
g\left(v, n^{g}(p)\right) & =g_{c}\left(v, n^{\mathcal{H}}(p)\right)+g\left(v, n^{\prime}(p)\right) \\
& =0
\end{aligned}
$$

where $n^{\prime}(p)=n^{g}(p)-n^{\mathcal{H}}(p)$ is orthogonal to $v$ since $g$ is an orthogonal extension. The last formula implies that $v \in T_{p} S$. So $T_{p}^{\mathcal{H}} S \subset \Delta_{p} \cap T_{p} S$. $\Delta_{p} \cap T_{p} S \subset T_{p}^{\mathcal{H}} S$ follows from the same argument.

REMARK 3.6. Therefore $T^{\mathcal{H}} S$ is the projection of $T S$ onto $\Delta$. In the case of Carnot groups of step two, if $p \in S$ is not a characteristic point, $T_{p}^{\mathcal{H}} S$ has obvious geometric meaning: $T_{p}^{\mathcal{H}} S$ is the projection on the horizontal bundle of the Lie algebra of the tangent group which is the blowup set of $S$ with respect to the natural dilations [that is the limit set of $S_{p, s}:=\delta_{1 / s}\left(p^{-1} S\right)$ under suitable topology as $s \rightarrow 0$ ], see [11]. This is the reason why we call $T_{p}^{\mathcal{H}} S$ the horizontal tangent plane.

If $p \in S \backslash \Sigma_{S}$, let $\mathcal{V}(p)=\left(n^{\mathcal{H}}(p)\right) /\left|n^{\mathcal{H}}(p)\right|$ be the unit horizontal normal. Then by Proposition $3, \mathcal{V}$ is intrinsic, independent of the choice of orthogonal extension, since $\Delta_{p}=T_{p}^{\mathcal{H}} S \stackrel{g_{c}}{\oplus} \mathcal{V}(p)$

LEMMA 3.7. Let $\mathcal{F}_{S}^{\Delta}$ be the set of all smooth vector fields tangent to $T^{\mathcal{H}} S$, that is,

$$
\mathcal{F}_{S}^{\Delta}:=\left\{f \in \Gamma(T S) \mid f(p) \in T_{p}^{\mathcal{H}} S \text { for any } p \in S\right\}
$$

Then $\mathcal{F}_{S}^{\Delta}$ is a projective $C^{\infty}(S)$-module. Here $C^{\infty}(S)$ denotes all smooth $\left(C^{\infty}\right)$ functions on $S$.

Proof: It is clear that $\mathcal{F}_{S}^{\Delta}$ is a $C^{\infty}$-submodule of $\Gamma(T S)$. To see it is also projective, let

$$
{ }^{\top} \mathcal{F}_{S}^{\Delta}:=\left\{f \in \Gamma(T S) \mid f(p) \notin T_{p}^{\mathcal{H}} S \text { for any } p \in S\right\} .
$$

Then ${ }^{\top} \mathcal{F}_{S}^{\Delta}$ is a $C^{\infty}(S)$-submodule of $\Gamma(T S)$ and by projecting (with respect to $g$ ) elements in $\Gamma(T S)$ to $\Delta$ we have

$$
\Gamma(T S)=^{\top} \mathcal{F}_{S}^{\Delta} \oplus \mathcal{F}_{S}^{\Delta}
$$

Since $\Gamma(T S)$ is a free $C^{\infty}(S)$-module, the statement follows from the last formula.

REMARK 3.8. Lemma 3.7 seemingly implies that $\mathcal{F}_{S}^{\Delta}$ is generated using an ordinary distribution using a Proposition in [32, p. 185] or [33, p. 11] where Vershik and Gershkovich asserted (but did not prove) that a differential system [a differential system is, 
by definition, a linear space of vector fields on a manifold that is a module over the ring of smooth functions] on a smooth manifold is a distribution if and only if it is a projective $C^{\infty}$-module. But the assertion is not true in general. For example, if $\Delta$ is of codimension one and $\Sigma_{S} \neq \emptyset$, then ${ }^{\top} \mathcal{F}_{S}^{\Delta}$ is by Lemma 3.7 a projective $C^{\infty}(S)$-module, but it is obviously not a distribution, since the set ${ }^{\top} \mathcal{F}_{S}^{\Delta}(p)$ of all evaluations at $p$ of vector fields in ${ }^{\top} \mathcal{F}_{S}^{\Delta}$ contains only one element (that is zero) if $p$ is a characteristic point, while ${ }^{\top} \mathcal{F}_{\mathcal{S}}^{\Delta}(p)$ is a subspace of one dimension if $p$ is not a characteristic point.

A natural question is whether there exist sufficiently many horizontal curves in hypersurfaces such that the intrinsic Carnot-Carathéodory metric can be defined. We shall not pursue the general case. But we shall prove that for contact manifolds of dimension more than three, there exists at least one smooth horizontal curve connecting two given points in a connected hypersurface $S$ if $\Sigma_{S}$ does not contain any immersed submanifold, and so the intrinsic Carnot-Carathéodory metric can be defined. This is the case if $S$ is noncharacteristic or with isolated characteristic points. Note that even in the contact case, the horizontal connectivity in hypersurfaces is difficult. Some authors asserted that there are few horizontal curves in hypersurfaces, even though these hypersurfaces are noncharacteristic, see for example, $[9$, p. 485$]$ where the authors wrote: “...Notice however that a $\mathrm{H}$-regular hypersurface contains very few $H$-rectifiable curves; in particular we cannot define a geodesic distance on a $H$-rectifiable hypersurface..." [smooth noncharacteristic hypersurfaces are, by definition, $H$-regular hypersurfaces; the inverse is not true in general. $H$-rectifiable curves are just absolutely continuous horizontal curves].

To prove Theorem 1.1, we first introduce the notion of the orbit of a family of vector fields.

Definition 3.9: (Orbits of a family of vector fields) Let $M$ be a connected smooth manifold and let $\mathcal{F}$ be any family of smooth vector fields globally defined on $M$. We define the orbit of a point $p \in M$ of this family as the set of points of $M$ reachable piececwise by trajectories of vector fields in the family, that is,

$$
\mathcal{O}_{p}:=\left\{\exp \left(t_{n} f_{k}\right) \circ \cdots \circ \exp \left(t_{1} f_{1}\right) \circ p \mid t_{i} \in \mathbb{R}, f_{i} \in \mathcal{F}, n \in \mathbb{N}\right\}
$$

where $\exp (t f)(p)$ denotes the flow of the vector field $f$ through $p$, that is, the curve $\gamma(t)$ in $M$ such that

$$
\left\{\begin{array}{l}
\dot{\gamma}(t)=f(\gamma(t)) \\
\gamma(0)=p
\end{array} .\right.
$$

Of course, if some of our vector fields are not complete then we consider only such $t_{1}, \ldots, t_{n}$ for which the above expression has sense. It is clear that the relation: " $q$ belongs to the orbit of $p$ " is an equivalence relation on $M$ and thus $M$ is the disjoint union of orbits (equivalence classes).

The following orbit theorem is due to Sussmann (also Nagano), see [25]. 
ThEOREM 3.10. (Orbit Theorem, Nagano-Sussmann) Let $\mathcal{F}$ be as above and let $p \in M$. Then:

(1) $\mathcal{O}_{p}$ is a connected immersion submanifold of $M$.

(2) $T_{q} \mathcal{O}_{p}=\operatorname{span}\left\{\left(P_{*} f\right)(q) \mid P \in \mathcal{P}, f \in \mathcal{F}\right\}, q \in \mathcal{O}_{p}$ where we denote by $\mathcal{P}$ the group of diffeomorphisms of $M$ generated by flows in $\mathcal{F}$ :

$$
\mathcal{P}=\left\{\exp \left(t_{n} f_{k}\right) \circ \cdots \circ \exp \left(t_{1} f_{1}\right) \mid t_{i} \in \mathbb{R}, f_{i} \in \mathcal{F}, n \in \mathbb{N}\right\} \subset \operatorname{Diff}(M)
$$

and by $P_{*}$ we mean the differential map of $P$.

REMARK 3.11. From Theorem 3.10, two simple but very useful observations are in order.

(1) First of all, if $f \in \mathcal{F}$, then $f(q) \in T_{q} \mathcal{O}_{p}$ for all $q \in \mathcal{O}_{p}$. Indeed, the trajectory $\exp (t f)(q)$ belongs to the orbit $\mathcal{O}_{p}$, thus its velocity vector $f(q)$ is in the tangent space $T_{q} \mathcal{O}_{p}$.

(2) Further, if $f_{1}, f_{2} \in \mathcal{F}$, then $\left[f_{1}, f_{2}\right](q) \in T_{q} \mathcal{O}_{p}$ for all $q \in \mathcal{O}_{p}$. This follows since the vector $\left[f_{1}, f_{2}\right](q)$ is tangent to the trajectory $\exp \left(-t f_{2}\right)$ $\circ \exp \left(-t f_{1}\right) \circ \exp \left(t f_{2}\right) \circ \exp \left(t f_{1}\right)(q) \in \mathcal{O}_{p}$. We go on and consider Lie brackets of arbitrarily high order

$$
\left[f_{1},\left[\ldots\left[f_{n-1}, f_{n}\right] \cdots\right]\right](q)
$$

as tangent vectors to $\mathcal{O}_{p}$ if $f_{i} \in \mathcal{F}$ and $q \in \mathcal{O}_{p}$.

These considerations can be summarised in terms of Lie algebra of vector fields generated by $\mathcal{F}$ :

$$
\text { Lie } \mathcal{F}:=\operatorname{span}\left\{\left[f_{1},\left[\ldots\left[f_{n-1}, f_{n}\right] \ldots\right]\right] \mid f_{i} \in \mathcal{F}, n \in \mathbb{N}\right\} \subset(\Gamma(T M))
$$

and its evaluation at a point $q \in M$ :

$$
\underset{q}{\operatorname{Lie}} \mathcal{F}=\{V(q) \mid V \in \operatorname{Lie} \mathcal{F}\} \subset T_{q} M
$$

We obtain the following statement.

Corollary 3.12 .

$$
\underset{q}{\operatorname{Lie}} \mathcal{F} \subset T_{q} \mathcal{O}_{p}
$$

for all $q \in \mathcal{O}_{p}$.

We note that the Chow connectivity theorem follows immediately from Corollary 3.12 .

Now we return to consider the horizontal connectivity in hypersurfaces in subRiemannian manifolds. We shall need the notion of horizontal immersed submanifold with respect to a distribution. 
DEFINITION 3.13: Let $M$ be a smooth manifold and $\Delta$ be a distribution on $M$. An immersed submanifold $i: N \rightarrow M$ of $M$ is horizontal with respect to $\Delta$ if $i_{*}\left(T_{p} N\right) \subset \Delta_{p}$ for any $p \in N$.

For a codimension one distribution we have

THEOREM 3.14. Let $M$ be a smooth connected manifold of dimension $m$ equipped with a smooth distribution $\Delta$ of dimension $m-1$. If $M$ does not admit horizontal immersion submanifolds of dimension $m-1$, then given two points $p, q$ in $M$, there exists at least one piecewise smooth horizontal (with respect to $\Delta$ ) curve connecting them (in fact $\Delta$ is equiregular).

Proof: Let $\mathcal{F}$ be the set of all smooth vector fields tangent to $\Delta$, that is,

$$
\mathcal{F}=\left\{f \in \Gamma(T M) \mid f(p) \in \Delta_{p} \text { for any } p \in M\right\} .
$$

For any $p \in M$ we claim that the orbit $\mathcal{O}_{p}$ of $p$ of the family $\mathcal{F}$ is of full dimension and thus $\mathcal{O}_{p}$ is an open set of $M$.

In fact, if not, then $\operatorname{dim}\left(\mathcal{O}_{p}\right) \leqslant m-1$ where $\operatorname{dim}\left(\mathcal{O}_{p}\right)$ denotes the dimension of $\mathcal{O}_{p}$. On the other hand by Corollary 3.12 , we have $\Delta_{q} \subset T_{q} \mathcal{O}_{p}$ for any $q \in \mathcal{O}_{p}$ and so the dimension of $\mathcal{O}_{p}$ is not less than $\operatorname{dim}(\Delta)=m-1$. Thus, $\operatorname{dim}\left(\mathcal{O}_{p}\right)=m-1$ and $T_{q} \mathcal{O}_{p}=\Delta_{q}$ for any $q \in \mathcal{O}_{p}$. So $\mathcal{O}_{p}$ is a horizontal immersed submanifold of dimension $m-1$. This contradicts with the assumption.

Since $M$ is connected and $M$ is the union of all orbits, we have $M=\mathcal{O}_{p}$ for any $p \in M$.

Lemma 3.15. Let $M$ be a smooth orientable contact manifold of dimension $2 n+1$ with a contact form $\eta$. Then $M$ does not admit horizontal (with respect to $\Delta=\operatorname{ker}(\eta)$ ) immersion submanifolds of dimension more than $n$.

PROOF: This is a well known fact. For the readers' convenience and completeness we give a proof.

Let $i: N \rightarrow M$ be a horizontal immersed submanifold of $M$ and let $p \in N$. By definition, $\operatorname{dim}\left(i_{*}\left(T_{p} N\right)\right)=\operatorname{dim}\left(T_{p} N\right)$ and for any $v_{1}, v_{2} \in i_{*}\left(T_{p} N\right)$,

$$
\omega_{i(p)}\left(v_{1}, v_{2}\right)=0
$$

where $\omega=d \eta$. Since $\omega_{i(p)}$ is a symplectic form on the horizontal space $\Delta_{p}$, from the non-degeneracy of $\omega_{i(p)}$ we have that

$$
\operatorname{dim}\left(\left(i_{*}\left(T_{p} N\right)\right)^{\perp}\right)+\operatorname{dim}\left(i_{*}\left(T_{p} N\right)\right)=2 n
$$

where $\left(i_{*}\left(T_{p} N\right)\right)^{\perp}$ is the symplectic orthogonal subspace of $i_{*}\left(T_{p} N\right)$. (3.1) is equivalent to $i_{*}\left(T_{p} N\right) \subset\left(i_{*}\left(T_{p} N\right)\right)^{\perp}$. Thus

$$
2 \operatorname{dim} i_{*}\left(T_{p} N\right) \leqslant \operatorname{dim}\left(\left(i_{*}\left(T_{p} N\right)\right)^{\perp}\right)+\operatorname{dim}\left(i_{*}\left(T_{p} N\right)\right)=2 n .
$$


Now we are in the position to prove Theorem 1.1.

ProOF of ThEOREM 1.1 Since $2 n-1>n$, the statement follows directly from Theorem 3.14 and Lemma 3.15 if $S$ is noncharacteristic. If $S$ possesses characteristic points, let $\mathcal{F}_{S}^{\Delta}$ be as in Lemma 3.7 and let $\mathcal{O}_{p}$ be an orbit of $p \in S$ of the family $\mathcal{F}_{S}^{\Delta}$. By Theorem $3.10 \mathcal{O}_{p}$ is an immersed submanifold. From the assumption that $\Sigma_{S}$ does not contain immersed submanifolds we conclude that there exists at least one point $q \in \mathcal{O}_{p}$ which is not a characteristic point. By Corollary 3.12 we have that $\mathcal{F}_{S}^{\Delta}(q) \subset T_{q}\left(\mathcal{O}_{p}\right)$ and the dimension of $\mathcal{O}_{p}$ is not less than $2 n-1=\operatorname{dim}\left(\mathcal{F}_{S}^{\Delta}(q)\right)$. Thus if $\operatorname{dim}\left(\mathcal{O}_{p}\right)=2 n-1$, then $\mathcal{O}_{p}$ is a horizontal immersion submanifold of $S$ (and $M$ ). This contradicts with Lemma 3.15 since $n>1$. So $\mathcal{O}_{p}$ is of full dimension and it is an open set of $S$. The assertion follows from the connectedness of $S$.

EXAMPLE 3.16. (The gauge ball in $\mathbb{H}^{n}$ with $n>1$ ) Let $\mathbb{S}^{n}=\left\{p \in \mathbb{R}^{2 n+1} \mid\|p\|=1\right\}$ where $\|p\|:=\left(\left(|x|^{2}+|y|^{2}\right)^{2}+|t|^{2}\right)^{1 / 4}$ is the gauge norm in $\mathbb{H}^{n}$. $\mathbb{S}^{n}$ is called the gauge ball centred in the origin. It is trivial to check that the metric induced by the gauge norm is left-invariant and 1-homogeneous with respect to natural dilations in $\mathbb{H}^{n}$. By direct computation the characteristic set $\Sigma_{\mathbb{S}^{n}}$ of $\mathbb{S}^{n}$ consists of only two points:

$$
\Sigma_{\mathbf{S}^{n}}=\{(0,0,1),(0,0,-1)\} \text {. }
$$

By Theorem 1.1, we see that the induced Carnot-Carathéodory metric $\mathbb{S}^{n}$ can be defined. EXAMPLE 3.17. (Hyperplanes in $\mathbb{H}^{n}$ with $n>1$ ) Let $n>1$. The vertical hyperplane $L_{i}=\left\{\left(x_{1}, \ldots, x_{i-1}, 0, x_{i+1}, \ldots, x_{n}, y, t\right) \in R^{2 n+1}\right\}$ of $\mathbb{H}^{n}$ is a Lie subgroup of $\mathbb{H}^{n}$ with the induced group law (that is the restriction to $L_{i}$ of the group law of $\mathbb{H}^{n}$ ) and Lie algebra $\mathcal{L}_{i}:=\bar{V}_{1} \oplus \bar{V}_{2}$ where

$$
\bar{V}_{1}=\operatorname{span}\left\{X_{1}, \ldots, X_{i-1}, X_{i+1}, \ldots, X_{n}, X_{n+1}, \ldots, X_{n+i-1}, \bar{X}_{n+i}, X_{n+i+1}, \ldots, X_{2 n}\right\}
$$

and $\bar{V}_{2}=\operatorname{span}\{T\}$ where $\bar{X}_{n+i}:=\frac{\partial}{\partial y_{i}}$ and $X_{i}, T$ as in Example 2.3). $L_{i}$ is noncharacteristic and is a Carnot group. It is easy to prove that the Carnot-Caratheodory metric $d_{\text {in }}$ induced by $\left(\bar{V}_{1}, g_{c}^{i}\right)$ where $g_{c}^{i}$ is the restriction of $g_{c}$ to $\bar{V}_{1}$ and the restricted CarnotCarathéodory metric $d_{\text {re }}$ satisfies that

$$
d_{\text {re }} \leqslant d_{\text {in }} \leqslant C d_{\text {re }}
$$

where $C$ is an absolute constant, see [16], and there are points $p, q \in L_{i}$ such that $d_{\mathrm{re}}(p, q)<d_{\mathrm{in}}(p, q)$.

The following example shows the condition of dimension more than three is unavoidable. 
EXAMPLE 3.18. In the simplest Heisenberg group $\mathbb{H}^{1}$, we consider horizontal curves in the horizontal hyperplane $L_{t}=\left\{(x, y, 0) \in \mathbb{R}^{3}\right\}$. Note that the point $(0,0,0)$ is the unique characteristic point in $L_{t}$. Let $\gamma(t)=(x(s), y(s), 0)$ be a horizontal curve in $L_{t}$. Then from (2.1) we have

$$
x(s) \dot{y}(s)=y(s) \dot{x}(s)
$$

and hence

$$
y(s)=C x(s)
$$

for some positive constant $C$. Thus there are no horizontal curves in any hypersurface $S \subset L_{1}$ which does not contain the point $(0,0,0)$. The same argument shows that there are no horizontal curves in $L_{x}=\left\{(0, y, t) \in \mathbb{R}^{3}\right\}$ and in $L_{y}=\left\{(x, 0, t) \in \mathbb{R}^{3}\right\}$.

REMARK 3.19. As in Euclidean geometry, $\mathbb{R}^{3}$ can serve as a model to the study of higher dimension, in the study of sub-Riemannian geometry $\mathbb{H}^{1}$ can also be seen as a model. But in developing geometric measure theory in the setting of sub-Riemannian geometry, in particular for the notion of rectifiability (and possibly for co-area formulae) in Carnot groups, $\mathbb{H}^{1}$ may be an exception. We recall that in $[9,10,11,12]$ Franchi, Serapioni and Serra Cassano have proposed a notion of rectifiability by introducing the notion of intrinsic regular hypersurfaces, and another notion which is a counterpart of Federer's definition of rectifiability where the "model spaces" are replaced by Carnot groups is announced by Pauls in [23]. It is obvious that the Paul's notion does not fit for $\mathbb{H}^{1}$. One reason is that the codimension one Lie subgroups $L_{x}, L_{y}$ of $\mathbb{H}^{1}$ have no stratified structure. Another reason is the horizontal non-connectivity of hypersurfaces in $\mathbb{H}^{1}$ as shown in the last example. A very intriguing question arises: we do not know whether the two notions of rectifiability for $\mathbb{H}^{n}(n>1)$ are equivalent in any reasonable sense.

\section{HoRizontal CONNECTION, THE hoRizontal MEAN CURVATURE AND THE HORIZONTAL DIVERGENCE THEOREM}

DEFINITION 4.1: (Horizontal connection) Let $g$ be any orthogonal extension of $g_{c}$ and let $\nabla$ be the Levi-Civita connection with respect to $g$. We define the horizontal connection $\widetilde{D}$ on $\Delta$ as

$$
\begin{gathered}
\widetilde{D}: \Gamma(\Delta) \times \Gamma(\Delta) \rightarrow \Gamma(\Delta) \\
\widetilde{D}_{X} Y=\sum_{i=1}^{k} g\left(\nabla_{X} Y, X_{i}\right) X_{i} \text { for any } X, Y \in \Gamma(\Delta)
\end{gathered}
$$

where $\left\{X_{1}, \ldots, X_{k}\right\}$ is an orthonormal basis of $\Delta$.

REMARK 4.2. The definition of $\widetilde{D}$ is independent of the choice of orthonormal basis of $\Delta$. In fact, let $\bar{X}_{i}=\sum_{j=1}^{k} a_{i j} X_{j}, i=1, \ldots, k$ be another orthonormal basis. Then $\left(a_{i j}\right)$ is 
an orthonormal matrix (everywhere) and hence

$$
\begin{aligned}
\sum_{i=1}^{k} g\left(\nabla_{X} Y, \bar{X}_{i}\right) \bar{X}_{i} & =\sum_{j=1}^{k} \sum_{l=1}^{k} \sum_{i=1}^{k} a_{i j} a_{i l} g\left(\nabla_{X} Y, X_{j}\right) X_{l}=\sum_{j=1}^{k} \sum_{l=1}^{k} \delta_{j l} g\left(\nabla_{X} Y, X_{j}\right) X_{l} \\
& =\sum_{j=1}^{k} g\left(\nabla_{X} Y, X_{j}\right) X_{j}
\end{aligned}
$$

Thus $\widetilde{D}$ is well defined.

We call $\widetilde{D}$ a "connection" because of the following fact.

LEMMA 4.3. $\widetilde{D}$ satisfies the following properties

(1) $\widetilde{D}_{X} Y$ is $R$-linear in both arguments,

(2) $\widetilde{D}_{X} Y$ is $C^{\infty}(M)$-linear in the argument of $X$,

(3) the Leibniz rule holds:

$\widetilde{D}_{X}(f Y)=(X f) Y+f \widetilde{D}_{X} Y$ for any $f \in C^{\infty}(M), X, Y \in \Gamma(\Delta)$

(4) $\widetilde{D}$ is compatible with respect to $g_{c}$, that is,

$$
X g_{c}(Y, Z)=g_{c}\left(\widetilde{D}_{X} Y, Z\right)+g_{c}\left(Y, \widetilde{D}_{X} Z\right) \text { for any } X, Y, Z \in \Gamma(\Delta),
$$

(5) the following "symmetry" property holds:

$$
\widetilde{D}_{X} Y-\widetilde{D}_{Y} X=[X, Y]^{\mathcal{H}} \text { for any } X, Y \in \Gamma(\Delta)
$$

where $[X, Y]^{\mathcal{H}}$ is, by definition, the projection of $[X, Y]$ to $\Delta$, that is,

$$
[X, Y]^{\mathcal{H}}=\sum_{i=1}^{k} g\left([X, Y], X_{i}\right) X_{i}
$$

Proof: The proof is trivial. All follow directly from the definition of $\widetilde{D}$, and the compatibility and symmetry of the Levi-Civita connection $\nabla$ together with the fact that $g$ is an orthogonal extension of $g_{c}$.

REMARK 4.4. For any vector field $X$, the horizontal part $X^{\mathcal{H}}$ of $X: X^{\mathcal{H}}=\sum_{i=1}^{k} g\left(X, X_{i}\right) X_{i}$, is independent of any orthogonal extension $g$ of $g_{c}$. In fact, since $g$ is an orthogonal extension of $g_{c}$, the projection, with respect to the decomposition of direct summation, of a vector field to the horizontal bundle is the same as the projection of this vector field to the horizontal bundle, with respect to the orthogonal decomposition.

From (2) and (3) of Lemma 4.3 it is straight to verify that $\widetilde{D}_{X} Y(p)$ depends only on $X(p)$ and the evaluations of $Y$ in a neighbourhood of $p$.

Any operator from $\Gamma(\Delta) \otimes \Gamma(\Delta) \rightarrow \Gamma(\Delta)$ satisfying (1), (2), (3) and (4) of Lemma 4.3 is called a nonholonomic connection. 
PRoposition 4.5. The operator $\widetilde{D}$ is independent of the choice of orthogonal extensions of $g_{c}$.

ProOF: From Lemma 4.3 we know that $\widetilde{D}_{U} V=\sum_{j=1}^{k} U\left(V^{j}\right) X_{j}+\sum_{i=1}^{k} \sum_{j=1}^{k} U^{i} V^{j} \widetilde{D}_{X_{i}} X_{j}$ for any $U=\sum_{i=1}^{k} U^{i} X_{i}, V=\sum_{j=1}^{k} V^{j} X_{j} \in \Gamma(\Delta)$. Thus $\widetilde{D}^{j=1}$ is determined by the connection coefficients $\Gamma_{i j}^{l}=g_{c}\left(\widetilde{D}_{X_{i}} X_{j}, X_{l}\right)=g\left(\nabla_{X_{i}} X_{j}, X_{l}\right)$. By the Cozhul's formula, for any $i, j, l=1, \ldots, k$ we have

$$
\begin{aligned}
g\left(\nabla_{X_{i}} X_{j}, X_{l}\right)= & \frac{1}{2}\left\{X_{i} g\left(X_{j}, X_{l}\right)+X_{j} g\left(X_{l}, X_{i}\right)-X_{l} g\left(X_{i}, X_{j}\right)\right. \\
& \left.-g\left(X_{i},\left[X_{l}, X_{j}\right]\right)-g\left(X_{j},\left[X_{i}, X_{l}\right]\right)+g\left(X_{l},\left[X_{j}, X_{i}\right]\right)\right\} \\
= & -\frac{1}{2}\left\{g\left(X_{i},\left[X_{l}, X_{j}\right]\right)+g\left(X_{j},\left[X_{i}, X_{l}\right]\right)-g\left(X_{l},\left[X_{j}, X_{i}\right]\right)\right\} \\
= & -\frac{1}{2}\left\{g_{c}\left(X_{i},\left[X_{l}, X_{j}\right]^{\mathcal{H}}\right)+g_{c}\left(X_{j},\left[X_{i}, X_{l}\right]^{\mathcal{H}}\right)-g_{c}\left(X_{l},\left[X_{j}, X_{i}\right]^{\mathcal{H}}\right)\right\}
\end{aligned}
$$

since $\left\{X_{1}, \ldots, X_{k}\right\}$ is an orthonormal basis of $\Delta$ with respect to $g_{c}$. The assertion follows from Remark 4.4.

Now we turn to the proof of Theorem 1.2. The definition of $D$ in Theorem 1.2 is inspired by the proof of Proposition 4.5 and the fundamental theorem in Riemannian geometry.

PROOF OF THEOREM 1.2 In this proof, to simplify the notations we use the Einstein summation convention: if in any term the same index name appears twice, as both an upper and a lower index, that term is assumed to be summed over all possible values of that index (from 1 to $k$ ). We first prove that $D$ is independent of the choice of orthonormal basis of $\Delta$. Let $\left\{\widetilde{X}_{1}, \ldots, \widetilde{X}_{k}\right\}$ be another orthonormal basis of $\Delta$, where $\tilde{X}_{i}=a_{i}^{j} X_{j}, i=1, \ldots, k$ and $A=\left(a_{i}^{j}\right)$ is an orthogonal matrix (everywhere). For any $U=\tilde{U}^{j} \tilde{X}_{j}=U^{j} X_{j}, V=\tilde{V}^{i} \tilde{X}_{i}=V^{i} X_{i} \in \Gamma(\Delta)$ where $\tilde{U}^{j}=b_{\tau}^{j} U^{r}, \tilde{V}^{i}=b_{s}^{i} V^{s}$ and $B=\left(b_{i}^{j}\right)$ is the inverse matrix of $A$ with $b_{i}^{j}=a_{j}^{i}$ for $i, j=1, \ldots, k$ since $A$ is orthogonal, we try to compute

$$
U\left(\tilde{V}^{i}\right) \widetilde{X}_{i}+\tilde{U}^{j} \tilde{V}^{i} \widetilde{\Gamma}_{i j}^{l} \widetilde{X}_{l}=\mathrm{I}+\mathrm{II}
$$

where $\tilde{\Gamma}_{i j}^{l}=-\left\{g_{c}\left(\widetilde{X}_{i},\left[\tilde{X}_{j}, \widetilde{X}_{l}\right]^{\mathcal{H}}\right)+g_{c}\left(\tilde{X}_{j},\left[\widetilde{X}_{l}, \widetilde{X}_{i}\right]^{\mathcal{H}}\right)-g_{c}\left(\tilde{X}_{l},\left[\tilde{X}_{i}, \tilde{X}_{j}\right]^{\mathcal{H}}\right)\right\} / 2$. Since

$$
\left[\tilde{X}_{j}, \tilde{X}_{l}\right]=a_{j}^{r} a_{l}^{h}\left[X_{r}, X_{h}\right]+a_{j}^{r} X_{r}\left(a_{l}^{h}\right) X_{h}-a_{l}^{h} X_{h}\left(a_{j}^{r}\right) X_{r}
$$

we have

$$
\left[\tilde{X}_{j}, \tilde{X}_{l}\right]^{\mathcal{H}}=a_{j}^{r} a_{l}^{h}\left[X_{r}, X_{h}\right]^{\mathcal{H}}+a_{j}^{r} X_{r}\left(a_{l}^{h}\right) X_{h}-a_{l}^{h} X_{h}\left(a_{j}^{r}\right) X_{r}
$$

and hence

$$
g_{c}\left(\tilde{X}_{i},\left[\tilde{X}_{j}, \tilde{X}_{l}\right]^{\mathcal{H}}\right)=a_{i}^{s} a_{j}^{r} a_{l}^{h} g_{c}\left(X_{s},\left[X_{r}, X_{h}\right]^{\mathcal{H}}\right)+\sum_{s=1}^{k} a_{i}^{s} a_{j}^{r} X_{r}\left(a_{l}^{s}\right)-\sum_{s=1}^{k} a_{i}^{s} a_{l}^{h} X_{h}\left(a_{j}^{s}\right),
$$


using the same arguments to other terms, and from $b_{i}^{j} a_{l}^{i}=\delta_{l}^{j}, a_{l}^{i} a_{l}^{j}=\delta_{i}^{j}$ for $i, j=1, \ldots, k$, we deduce

$$
\begin{aligned}
\mathrm{II}=- & \frac{1}{2} \sum_{h=1}^{k} V^{s} U^{r}\left\{g_{c}\left(X_{s},\left[X_{r}, X_{h}\right]^{\mathcal{H}}\right)+g_{c}\left(X_{r},\left[X_{h}, X_{s}\right]^{\mathcal{H}}\right)-g_{c}\left(X_{h},\left[X_{s}, X_{r}\right]^{\mathcal{H}}\right)\right\} X_{h} \\
- & \frac{1}{2}\left\{\sum_{s=1}^{k} \sum_{l=1}^{k} U^{r} V^{s} a_{l}^{h} X_{r}\left(a_{l}^{s}\right) X_{h}-\sum_{s=1}^{k} \sum_{h=1}^{k} U^{r} V^{s} b_{r}^{j} X_{h}\left(a_{j}^{s}\right) X_{h}-U^{r} V^{s} b_{r}^{j} X_{s}\left(a_{j}^{h}\right) X_{h}\right. \\
& \left.+U^{r} V^{s} b_{s}^{i} X_{r}\left(a_{i}^{h}\right) X_{h}-\sum_{r=1}^{k} \sum_{h=1}^{k} U^{r} V^{s} b_{s}^{i} X_{h}\left(a_{i}^{r}\right) X_{h}+\sum_{r=1}^{k} \sum_{l=1}^{k} U^{r} V^{s} a_{l}^{h} X_{s}\left(a_{l}^{r}\right) X_{h}\right\} .
\end{aligned}
$$

Renaming and rearranging some indices, then cancelling or adding some terms (using $X_{l}\left(a_{r}^{j} b_{j}^{h}\right)=0$ and $a_{l}^{r}=b_{r}^{l}$ for any $\left.l, r, h=1, \ldots, k\right)$, we infer that

$$
\mathrm{II}=U^{r} V^{s} \Gamma_{s r}^{h} X_{h}-U^{r} V^{j} a_{i}^{l} X_{r}\left(b_{j}^{i}\right) X_{l} .
$$

Since

we obtain

$$
\begin{aligned}
\mathrm{I} & =U\left(\tilde{V}^{j}\right) \tilde{X}_{j}=U^{r} X_{r}\left(b_{i}^{j} V^{i}\right) a_{j}^{l} X_{l} \\
& =U^{r} X_{r}\left(V^{i}\right) b_{i}^{j} a_{j}^{l} X_{l}+U^{r} V^{i} X_{r}\left(b_{i}^{j}\right) X_{l} \\
& =U\left(V^{j}\right) X_{j}+U^{r} V^{i} X_{r}\left(b_{i}^{j}\right) a_{j}^{l} X_{l},
\end{aligned}
$$

$$
U\left(\tilde{V}^{i}\right) \tilde{X}_{i}+\tilde{U}^{j} \tilde{V}^{i} \tilde{\Gamma}_{i j}^{l} \widetilde{X}_{l}=U\left(V^{j}\right) X_{j}+U^{r} V^{s} \Gamma_{s r}^{h} X_{h}
$$

Thus $D$ is well defined.

From the definition of $D$ we have for any $i, j, l=1, \ldots, l$,

$$
\begin{gathered}
\Gamma_{i j}^{l}=g_{c}\left(D_{X_{j}} X_{i}, X_{i}\right), \\
\Gamma_{j i}^{l}+\Gamma_{l i}^{j}=0, \\
\Gamma_{i j}^{l}-\Gamma_{j i}^{l}=g_{c}\left(\left[X_{j}, X_{i}\right]^{\mathcal{H}}, X_{l}\right) .
\end{gathered}
$$

Properties (1)-(3) are obvious. To prove (4), let $U=U^{j} X_{j}, V=V^{i} X_{i}$, $W=W^{l} X_{l}$. Then from (2) and (3) we have

$$
\begin{aligned}
g_{c}\left(D_{V} U, W\right)+g_{c}\left(U, D_{V} W\right) & =U^{j} W^{l} g_{c}\left(D_{X_{j}}\left(V^{i} X_{i}\right), X_{l}\right)+U^{j} V^{i} g_{c}\left(X_{j}, D_{X_{\mathrm{i}}}\left(W^{l} X_{l}\right)\right) \\
& =U^{j} V^{i} W^{l}\left(g_{c}\left(D_{X_{j}} X_{i}, X_{l}\right)+g_{c}\left(X_{j}, D_{X_{\mathrm{i}}} X_{l}\right)\right)+\sum_{i=1}^{k} W^{i} V\left(U^{i}\right) \\
& +\sum_{j=1}^{k} U^{j} V\left(W^{j}\right) \\
& =U^{j} V^{i} W^{l}\left(g_{c}\left(D_{X_{j}} X_{i}, X_{l}\right)+g_{c}\left(X_{j}, D_{X_{i}} X_{l}\right)\right)+V g_{c}(W, U) .
\end{aligned}
$$

Since by (4.3) and (4)

$$
\begin{aligned}
g_{c}\left(D_{X_{j}} X_{i}, X_{l}\right)+g_{c}\left(X_{j}, D_{X_{i}} X_{l}\right) & =\Gamma_{j i}^{l}+\Gamma_{l j}^{i} \\
& =0
\end{aligned}
$$


for any $i, j, l=1, \ldots, k$, we conclude that

$$
g_{c}\left(D_{V} U, W\right)+g_{c}\left(U, D_{V} W\right)=V g_{c}(W, U) .
$$

To see (5), for any $l=1, \ldots, k, U=U^{j} X_{j}, V=V^{i} X_{i} \in \Gamma(\Delta)$, by (4.5) we have

$$
\begin{aligned}
g_{c}\left(D_{U} V-D_{V} U, X_{l}\right) & =U\left(V^{l}\right)-V\left(U^{l}\right)+U^{j} V^{i}\left(\Gamma_{i j}^{l}-\Gamma_{j i}^{l}\right) \\
& =U\left(V^{l}\right)-V\left(U^{l}\right)+U^{j} V^{i} g_{c}\left(\left[X_{j}, X_{i}\right]^{\mathcal{H}}, X_{l}\right) \\
& =g_{c}\left([U, V]^{\mathcal{H}}, X_{l}\right) .
\end{aligned}
$$

The uniqueness follows from an argument similar to the Riemannian case: first use (2) and (3) to obtain (1.1) where $\Gamma_{i j}^{l}=g_{c}\left(D_{X_{j}} X_{i}, X_{l}\right)$, then using (1.2) and (1.3) to get a formula of Cozhul type,

$$
g_{c}\left(D_{X_{j}} X_{i}, X_{l}\right)=-\frac{1}{2}\left\{g_{c}\left(X_{i},\left[X_{l}, X_{j}\right]^{\mathcal{H}}\right)+g_{c}\left(X_{j},\left[X_{i}, X_{l}\right]^{\mathcal{H}}\right)-g_{c}\left(X_{l},\left[X_{j}, X_{i}\right]^{\mathcal{H}}\right)\right\} .
$$

REMARK 4.6. Thus $D$ is an intrinsic notion of the sub-Riemannian structure $\left(\Delta, g_{c}\right)$. Here we must point out that the connection $\bar{D}$ obtained by projecting the Levi-Civita connection onto the horizontal bundle was first introduced by Schouten in [24]. (There the Riemannian metric $g$ with respect to which the Levi-Civita connection was computed was not necessarily an orthogonal extension of $g_{c}$. Thus in this case $\bar{D}$ is not intrinsic: it depends on 9 .) It was further developed by Vagner in $[27,28,29,30]$ (see [15] for a survey), and also $[31,32]$ where $\bar{D}$ is called "truncated connection". But it seems that they did not prove the existence of $D$ in Theorem 1.2 and its intrinsic nature (determination by $\left.\left(\Delta, g_{c}\right)\right)$ of $D$, see $[32$, p. 202].

In Russian mathematicians' papers (also Cartan's, see $[3,2,17]$ ) nonholonomic connection was mainly used to study "geodesics" (but not sub-Riemannian geodesics, that is, nonholonomic geodesics) and curvature of distributions in the setting of nonholonomic dynamic systems. Let $\gamma(s)$ be a smooth horizontal curve in $M$. We call $\gamma$ a "geodesic" if $D_{\dot{\gamma}} \dot{\gamma}=0$. The mechanical significance of such "geodesics" lies in they characterise the trajectories of motion of a mechanical system with quadratic Lagrangian and linear constraints (say $\Delta$ ), see $[31,32]$ for details.

EXAMPLE 4.7. (The horizontal connection in a Carnot group) For a Carnot group $G$, since its Lie algebra $\mathcal{G}$ is graded, if we choose a system of left invariant vector fields $\left\{X_{1}, \ldots, X_{k}\right\}$ as an orthogonal basis of the horizontal bundle $\Delta=V_{1}$, then the connection coefficients $\Gamma_{i j}^{l}$ of the horizontal connection $D$ are vanishing everywhere for any $i, j, l$ $=1, \ldots, k$. For this basis, $D$ has the simple form

$$
D_{U} V=\sum_{i=1}^{k} U\left(V^{i}\right) X_{i} \text { for any } U, V=\sum_{i=1}^{k} V^{i} X_{i} \in \Gamma(\Delta),
$$

see [26] for details. 
For completeness we introduce the notion of horizontal divergence.

Definition 4.8: (Horizontal divergence) Let $X \in \Gamma(\Delta)$. The horizontal divergence $\operatorname{div}_{\mathcal{H}} X$ of $X$ is defined by

$$
\operatorname{div}_{\mathcal{H}} X=\sum_{i=1}^{k} g_{c}\left(D_{X_{i}} X, X_{i}\right)
$$

Note that as shown in Remark 4.2, $\operatorname{div}_{\mathcal{H}} X$ is independent of the choice of orthonormal basis of $\Delta$ because of (2) in Lemma 4.3 .

The following proposition, which in the case of Carnot groups is well known, follows immediately from the definitions.

Proposition 4.9. $\operatorname{div}_{\mathcal{H}} X=\operatorname{div} X$ for any $X \in \Gamma(\Delta)$ where $\operatorname{div} X$ is the usual divergence of $X$ computed with respect to any orthogonal extension $g$ of $g_{c}$.

It is interesting that $D$ can be used to define the horizontal mean curvature of (noncharacteristic) hypersurfaces in $\left(M, \Delta, g_{c}\right)$. In the rest of this paper, we discuss how this can be done. Roughly speaking, in sub-Riemannian (or nonholonomic) geometry horizontal connection, horizontal tangent connection, horizontal normal, horizontal second fundamental form and horizontal mean curvature are counterparts of Levi-Civita connection, tangent connection, Riemannian normal, second fundamental form and mean curvature in Riemannian geometry, respectively.

We assume, if without further notice, $S$ is a smooth noncharacteristic hypersurface in a sub-Riemannian manifold $\left(M, \Delta, g_{c}\right)$. Then $T^{\mathcal{H}} S$ is a subbundle of $T S$ of dimension $k-1$. From the definition we have

Lemma 4.10. If $X, Y \in \Gamma\left(T^{\mathcal{H}} S\right)$, then $[X, Y]^{\mathcal{H}} \in \Gamma\left(T^{\mathcal{H}} S\right)$.

It is clear that any vector $v$ in $T_{p}^{\mathcal{H}} S(p \in S)$ can be extended to a vector field in $T^{\mathcal{H}} S$ by first extending $v$ to a vector field $V$ in $T S$ then projecting $V$ to $T^{\mathcal{H}} S$, and any vector field $V$ in $T^{\mathcal{H}} S$ can smoothly extended to a horizontal vector field in $\Delta$ by first extending $V$ to a vector field $\bar{V}$ in $T M$ then projecting $\bar{V}$ to $\Delta$. Sometimes we shall denote by the same symbol both the extended vector field and the original vector field, in particular when we have confirmed that the objects under consideration are independent of such extensions.

If $X, Y$ are vector fields in $T^{\mathcal{H}} S$, we can extend them to horizontal vector fields $\bar{X}, \bar{Y}$ in $M$, apply the ambient derivative operator $D$, and then decompose at points of $S$ to get

$$
D_{\bar{X}} \bar{Y}(x)=\left(D_{\bar{X}} \bar{Y}\right)^{\top}(x)+\left(D_{\bar{X}} \bar{Y}\right)^{\perp}(x) \quad x \in S
$$

where $\left(D_{\bar{X}} \bar{Y}\right)^{\top}(x),\left(D_{\bar{X}} \bar{Y}\right)^{\perp}(x)$ are the projections of $D_{\bar{X}} \bar{Y}(x)$ onto $T_{x}^{\mathcal{H}} S$ and the direction of $\mathcal{V}(x)$ respectively, where $\mathcal{V}(x)$ is the unit horizonal normal, see Remark 3.6. 
Definition 4.11: (The horizontal second fundamental form) Let $X, Y$ be vector fields in $T^{\mathcal{H}} S$. We define

$$
\mathrm{II}(X, Y):=\left(D_{\bar{X}} \bar{Y}\right)^{\perp}
$$

where $\bar{X}, \bar{Y}$ are the arbitrarily extended horizontal vector fields of $X, Y$ respectively, as the horizontal second fundamental form of $S$.

THEOREM 4.12. The horizontal second fundamental form $I I(X, Y)$ is

(1) independent of the extension of $X$ and $Y$;

(2) bilinear over $C^{\infty}(S)$; and

(3) symmetric in $X$ and $Y$.

The proof of Theorem 4.12 is very similar to the Riemannian case, see [26]. Note that (4.12) follows from Lemma 4.10 and the symmetry property (4.2) of $D$, as in the Riemannian geometry the symmetry of the second fundamental form follows directly from the symmetry (torsion free) of the Levi-Civita connection. Theorem 4.12 in particular implies that $\mathrm{II}(X, Y)(p)$ depends only on $X(p)$ and $Y(p)$.

DEFINITION 4.13: (The horizontal tangent connection) We define the horizontal tangent connection

$$
D^{\top}: \Gamma\left(T^{\mathcal{H}} S\right) \otimes \Gamma\left(T^{\mathcal{H}} S\right) \rightarrow \Gamma\left(T^{\mathcal{H}} S\right)
$$

by

$$
D_{X}^{\top} Y=\left(D_{\bar{X}} \bar{Y}\right)^{\top}
$$

where $\bar{X}, \bar{Y}$ are the arbitrarily extended horizontal vector fields of $X, Y$ respectively.

THEOREM 4.14. The horizontal tangent connection $D^{\top}$ is well defined, that is, $D_{X}^{\top} Y$ is independent of the extension of $X$ and $Y$. Moreover $D^{\top}$ satisfies (1)-(5) of Lemma 4.3 where $\Delta$ is replaced by $T^{\mathcal{H}} S$ and $g_{c}$ is replaced by $g_{c}^{\mathcal{H}}$ which is the restriction of $g_{c}$ to $T^{\mathcal{H}} S$.

The proof of Theorem 4.14 is direct and trivial. From the preceding discussions, we know that in $S$ there exists an intrinsic horizontal connection determined by the subRiemannian structure $\left(T^{\mathcal{H}} S, g_{c}^{\mathcal{H}}\right)$, since

$$
T S=T^{\mathcal{H}} S \stackrel{\bar{g}}{\bigoplus}\left(T S / T^{\mathcal{H}} S\right)
$$

where $\bar{g}$ is the restriction of $g$ to $T S$, see the proof of Lemma 3.7. From the definitions it is easy to see that $\bar{g}$ is the orthogonal extension of $g_{c}^{\mathcal{H}}$, even if $T^{\mathcal{H}} S$ may not satisfy the Chow condition. Thus the "external" connection $D^{\top}$ is equal to the intrinsic one by Theorem 4.14 and Theorem 1.2.

Definition 4.15: (Horizontal tangent divergence) Let $X \in \Gamma\left(T^{\mathcal{H}} S\right)$. The horizontal tangent divergence $\operatorname{div}_{\mathcal{H}}^{\top} X$ of $X$ is defined by

$$
\operatorname{div}_{\mathcal{H}}^{\top} X=\sum_{i=1}^{k-1} g_{c}^{\mathcal{H}}\left(D_{\tau_{i}}^{\top} X, \tau_{i}\right)
$$


where $\left\{\tau_{1}, \ldots, \tau_{k-1}\right\}$ is a orthonormal basis of $T^{\mathcal{H}} S$ with respect to $g_{c}^{\mathcal{H}}$. For a horizontal vector field $Y$ defined in $S$, that is, $Y \in \Gamma\left(\left.\Delta\right|_{S}\right)$ where $\left.\Delta\right|_{S}$ is the restriction of $\Delta$ to $S$, we can also define its horizontal tangent divergence

$$
\operatorname{div}_{\mathcal{H}}^{\top} Y=\sum_{i=1}^{k-1} g_{c}\left(D_{\tau_{i}} X, \tau_{i}\right)
$$

Similarly to Proposition 4.9 , we have

Proposition 4.16. Let $Y \in \Gamma\left(T^{\mathcal{H}} S\right)$. Then

$$
\operatorname{div}_{\mathcal{H}}^{\top} Y=\operatorname{div}_{S} Y
$$

where $\operatorname{div}_{S} Y$ is the Riemannian tangent divergence of $Y$ on $S$ computed with respect to any orthogonal extension $g$ of $g_{c}$.

Definition 4.17: The scalar horizontal second fundamental form $h$ is the symmetric bilinear function on $T^{\mathcal{H}} S$ defined by

$$
h(X, Y)=g_{c}(\mathrm{II}(X, Y), \mathcal{V}) .
$$

That is $\operatorname{II}(X, Y)=h(X, Y) \mathcal{V}$. Thus $h$ uniquely determines an endomorphism of $T^{\mathcal{H}} S$, say $A$, that is,

$$
g_{c}(A X, Y)=h(X, Y) \quad \text { for all } X, Y \in \Gamma\left(T^{\mathcal{H}} S\right) \text {. }
$$

$A$ is self-adjoint and we call $A$ the horizontal shape operator of $S$.

For any $p \in S, A$ gives a symmetric linear map $A_{p}: T_{p}^{\mathcal{H}} S \rightarrow T_{p}^{\mathcal{H}} S$. Then by the symmetry of $A_{p}, A_{p}$ has $k-1$ real eigenvalues.

DEFinition 4.18: (The horizontal mean curvature) The $k-1$ eigenvalues of $A_{p}$, $\kappa_{1}, \ldots, \kappa_{k-1}$, are called the horizontal principal curvatures at $p$ and the corresponding eigenspaces are called horizontal principal directions. We define the horizontal mean curvature $H_{X}(p)$ at $p$ the trace of $A_{p}$, that is, $H_{X}(p)=\sum_{i=1}^{k-1} \kappa_{i}$ and call the product of $\kappa_{1}, \ldots, \kappa_{k-1}$ the horizontal Gaussian curvature at $p$.

It has been proved in [26] that in the case of Carnot groups our definition of the horizontal mean curvature coincides with that in [6] and [22].

REMARK 4.19. Since $D, T^{\mathcal{H}} S$ and $\mathcal{V}$ are intrinsic, so is the notion of horizontal mean curvature. As in [6] and [22], the horizontal mean curvature can be defined only at noncharacteristic points [in [6] (see also [14]), if $p$ is a characteristic point, the horizontal mean curvature at $p$ is defined by $H(p)=\lim _{q \rightarrow p} H(q)$ if this limit exists. Since it is impossible to determine the size of the set of characteristic points at which the limits exist, this definition seems meaningless]. However by Proposition 3.2, the horizontal mean curvature can be defined almost everywhere. 
EXAMPLE 4.20. (Horizontal mean curvature of noncharacteristic hypersurfaces in Carnot groups) As in Example 4.7, choose a system of left-invariant vector fields $\left\{X_{1}, \ldots, X_{k}\right\}$ as an orthogonal basis of the horizontal bundle, then for any noncharacteristic hypersurface $S \subset G$, the horizontal mean curvature can be expressed as the form

$$
H(p)=\sum_{i=1}^{k} X_{i}\left(\mathcal{V}^{i}\right)(p) \text { for any } p \in S
$$

where $\mathcal{V}=\sum_{i=1}^{k} \mathcal{V}^{i} X_{i}$ is the unit horizontal normal of $S$, see [26].

\section{REFERENCES}

[1] A. Bellaïche, 'Tangent spaces in sub-Riemannian geometry', in Sub-Riemannian Geometry, (A. Bellaïche and J.J. Risler, Editors), Progress in Math. 144 (Birkhäuser, Basel, 1996), pp. 1-78.

[2] A.M. Bloch, P.S. Krishnaprasad, J.E. Marsden and R.M. Murray, 'Nonholonomic mechanical systems with symmetry', Arch. Rational Mech. Anal. 136 (1996), 21-99.

[3] E. Cartan, 'Sur la representation geometrique des syst'emes materiels non holonomes', Proc. Int. Congr. Math. 4 (1928), 253-261.

[4] W.L. Chow, 'über Systeme non linearen partiellen Differentialgleichungen erster Ordung', Math. Ann. 117 (1939), 98-105.

[5] D. Danielli, N. Garofalo and D.M. Nhien, 'Minimal surfaces, surfaces of constant mean curvature and isoperimetry in Carnot groups', (preprint).

[6] D. Danielli, N. Garofalo and D.M. Nhien, 'Non-doubling Ahlfors measures, perimeter measures, and the characterization of the trace spaces of Sobolev functions in Carnot-Carathéodory spaces', Mem. Amer. Math. Soc. (to appear).

[7] M. Derridj, 'Un probléme aux limites pour une classe d'opérateurs du second ordre hypoelliptiques', Ann. Inst. Fourier (Grenoble) 21 (1971), 99-148.

[8] M. Derridj, 'Sur un théorème de traces', Ann. Inst. Fourier (Grenoble) 22 (1972), 73-83.

[9] B. Franchi, R. Serapioni and F. Serra Cassano, 'Rectifiability and perimeter in the Heisenberg group', Math. Ann. 321 (2001), 479-531.

[10] B. Franchi, R. Serapioni and F. Serra Cassano, 'Regular hypersurfaces, intrinsic perimeter and implicit functions theorem in Carnot groups', Comm. Anal. Geom. (2003), 909-944.

[11] B. Franchi, R. Serapioni and F. Serra Cassano, 'On the structure of finite perimeter sets in step 2 Carnot groups', (preprint available from http://cvgmt.sns.it).

[12] B. Franchi, R. Serapioni and F. Serra Cassano, 'Rectifiability and perimeter in step 2 groups', (preprint available from http://cvgmt.sns.it).

[13] N. Garofalo and D.M. Nhieu, 'Isoperimetric and Sobolev inequalities for Carnot-Carathéodory spaces and the existence of minimimal surfaces', Comm. Pure Appl. Math. 49 (1996), 1081-1144.

[14] N. Garofalo and S.D. Pauls, 'The Bernstein problem in the Heisenberg group', (preprint available from http://cvgmt.sns.it).

[15] E.M. Gorbatenko, 'Differential geometry of nonholonomic manifolds (after V.V.Vagner)', Ukrain. Geom. Sb. (1985), 31-43. 
[16] M. Gromov, 'Carnot-Carathéodory spaces seen from within', in Sub-Riemannian Geometry, (by A. Bellaïche and J.J. Risler, Editors), Progress in Math. 144 (Birkhäuser, Basel, 1996), pp. 79-323.

[17] J. Koiller, P.R. Rodrigues and P. Pitanga, 'Non-holonomic connections following Élie Cartan', An. Acad. Bras. Cienc. 7 (2001), 165-190.

[18] U. Koschorke, Vector fields and other vector bundle morphisms-a singularity approach, Lecture notes in Mathematics 847 (Springer-Verlag, Berlin, 1981).

[19] V. Magnani, Elements of geometric measure theory on sub-Riemannian groups, Ph.D. thesis, Scuola Normale Superiore, Pisa, 2002.

[20] V. Magnani, 'A blow-up theorem for regular hypersurfaces on nilpotent groups', Manuscripta Math. 110 (2003), 55-76.

[21] R. Montgomery, A tour of Subriemannian geometry, their geodesics and applications, Mathematical Surveys and Monographs 91 (American Mathematical Society, Providence, R.I., 2002).

[22] S.D. Pauls, 'Nonparametric minimal surfaces in the Heisenberg group', Geometriae Dedicata (to appear).

[23] S.D. Pauls, 'A notion of rectifiability modelled on Carnot groups', Indiana Univ. Math. J. 53 (2004), 49-81.

[24] J.A. Schouten, 'On nonholonomic connections', Proc. Amsterdam Nederl. Akad. Wetensch. Ser. A, 31 (1928), 291-299.

[25] H.J. Sussmann, 'Orbits of families of vector fields and integrability of distributions', Trans. Amer. Math. Soc. 80 (1973), 171-188.

[26] K.H. Tan and X.P. Yang, 'Horizontal connection and horizontal mean curvature in Carnot groups', (preprint).

[27] V.V. Vagner, 'Differential geometry of nonholonomic manifolds', (in Russian), Tr. Semin. Vectorn. Tenzorn. Anal. 213 (1935), 269-314.

[28] V.V. Vagner, 'Differential geometry of nonholonomic manifolds', in The VIII-th International Competition for the N. I. Lobatschewski Prize Report (The Kazan Physico-Mathematical Society, Kazan, 1940).

[29] V.V. Vagner, 'A geometric interpretation of nonholonomic dynamical systems', Tr. Semin. Vectorn. Tenzorn. Anal. 5 (1941), 301-327.

[30] V.V. Vagner, Geometria del calcolo delle variazioni 2 (C.I.M.E., Roma).

[31] A.M. Vershik and L.D. Faddeev, 'Lagrange mechanics in an invariant form', in Probl. Theor. Phys. Vol.II, pp. 129-141. English translation: Sel. Math. Sov. 1 (1981), 339350.

[32] A.M. Vershik and V.Ya. Gershkovich, 'Nonholomic problems and the theory of distributions', Acta. Appl. Math. 12 (1988), 181-209.

[33] A.M. Vershik and V.Ya. Gershkovich, 'Nonholonomic dynamical systems, geometry of distributions and variational problems', in Dynamical Systems VII, (V.I. Arnold and S.P. Novikov, Editors), Encyclopaedia of Mathematics Sciences 16 (Springer-Verlag, 1994).

Department of Applied Mathematics

Nanjing University of Science and Technology

210094, Nanjing, Jiangsu

Peoples Republic of China

e-mail: tankanghai2000@yahoo.com.cn yangxp@mail.njust.edu.cn 